Calderón García, Natalia.

Universidad de Barcelona, en su caso: estudiante de doctorado, Universidad de Barcelona, Pedagogías Culturales.

\title{
La Investigación Artística como espacio disruptivo de lo artístico y lo pedagógico
}

\author{
TIPO DE TRABAJO \\ Comunicación. \\ PALABRAS CLAVE \\ Investigación Artística, producción de conocimiento, lo pedagógico, des-conocido.
}

KEY WORDS

Artistic Research, knowledge production, the pedagogical, un-known.

\section{RESUMEN}

En mi tesis doctoral Irrumpir lo artístico, perturbar lo pedagógico: La Investigación Artística como espacio social de producción de conocimiento (2015) utilizo como herramientas de investigación, los medios audiovisuales para registrar un encuentro dibujado donde la artista (Núria Güell), el comisario (Oriol Fontdevila) y la investigadora (yo), reflexionamos juntos sobre los modos de conocer en torno a la producción de un proyecto artístico.

Durante la edición del vídeo En colaboración y conflicto. El trazo de nueve modos de conocer, desvele nueve modos por los que el proyecto produjo conocimientos.

A partir de estos modos de conocer me pregunto ¿Qué espacio social se construyó mediante estas relaciones de producción de conocimiento?: ¿Cómo pueden estos modos de conocer informar sobre las nuevas posibilidades que abre la Investigación Artística? ¿Cómo se modifica lo artístico y lo pedagógico al analizarlos como relaciones espaciales, sociales y políticas que constituyen espacios de saber y de ser?

La maquinaria epistemológica que significa y da valor a las significaciones que construimos en el proyecto La Síndrome de Sherwood ha de sido puesta en cuestión con dos representaciones principales: el texto y los vídeos. Estas representaciones fueron construidas desde la reflexividad para hacer engarzar las piezas de distintas formas y a través de un posicionamiento disruptivo que alteró mi relación respecto a la Investigación Artística, lo pedagógico y a lo artístico.

\section{ABSTRACT}

In my dissertation Irrumpir lo artístico, perturbar lo pedagógico: La Investigación Artística como espacio social de producción de conocimiento (2015) I make use of research tools such as audiovisual media to record a drawing encounter where the artist (Núria Güell), the curator (Oriol Fontdevila) and the researcher (me) reflected together about the ways of knowing around an art project production.

During the edition of the video: En colaboración y conflicto. El trazo de nueve modos de conocer, I unveiled nine ways through which the project produced knowledge. And, based on these ways of knowing I ask myself: What social space was built through these relations of knowledge production? How can these ways of knowing inform about the new possibilities 
Artistic Research opens? How are the artistic and the pedagogical modified when are being analyzed as spatial, social and political relationships that constitute being and knowing spaces?

Two main representations, the text of my dissertation and the videos that take part of it, question the epistemological machinery that signifies and values the meanings of the project La Sindrome de Sherwood. I built these representations using reflexivity to entangle pieces of different forms and through a disruptive position that altered my relation towards Artistic Research, the pedagogical and the artistic.

\section{CONTENIDO}

\section{Introducción}

Durante los cuatro años que he dedicado a mi investigación doctoral, enfoqué mis intereses hacia responder qué conocimientos resultaban de la realización de un proyecto artístico: La síndrome de Sherwood de la artista Núria Güell y el comisario Oriol Fontdevila. Para poder visibilizar los conocimientos que el proyecto había construido me concentré en trazar las relaciones epistemológicas y con ellas, los espacios sociales que habían generado.

Mi investigación abrió un espacio para nuevas relaciones epistemológicas y ontológicas al desestabilizar la práctica artística y, al ponerla en tensión a través de la noción de lo pedagógico, dejar de verla como ilustradora o productora de artefactos. Es decir que no sólo busqué visibilizar los aprendizajes producidos en el proyecto artístico, sino que reflexioné acerca de cómo se generaron éstos y qué nuevos sujetos pedagógicos -subjects-yet-to-come- ${ }^{1}$ se conformaron en este proceso.

Para poder dar forma a relaciones que delimitaron el espacio social de producción de conocimiento, propuse a la artista y al comisario un encuentro en el que les pedí que trazaran los agentes involucrados en su proyecto y la manera en que habían vinculado éstos. El encuentro -los dibujos y las conversaciones- fue registrado en vídeo con cuatro distintas cámaras que pusieron de manifiesto mi posicionamiento de perspectiva múltiple, que, de la mano del Construccionismo social ${ }^{2}$, es reflejo de una visión parcial y fragmentada.

A partir de la edición de los vídeos encontré en las reflexiones de Núria y Oriol nueve modos de conocer que pude reconocer a partir de estrategias de codificación inspiradas en los modos de análisis de la Grounded Theory de la segunda generación ${ }^{3}$.

La Investigación Artística que permite y promueve el uso de herramientas artísticas (el vídeo y el dibujo, en este caso) para la investigación, fue la columna vertebral de mi proceso metodológico. Al ser éste un camino en construcción, que no es definido a priori sino en el proceso mismo, fue indispensable dar cuenta de la toma de decisiones según la reflexividad ${ }^{4}$. La reflexividad demanda del investigador/investigadora el cuestionamiento de su propia interpretación, Macbeth lo llama investigador en constante autoinspección (researcher in ongoing self-scrutiny).

La comunicación que a continuación presento trata de limitarse a una parte de mi investigación que cuestiona el espacio disruptivo entre lo artístico y lo pedagógico, y genera nuevos vínculos entre la academia y el arte para posibilitar nuevos espacios de conocimiento.

\section{Desarrollo}

¿Qué espacio se abre entre lo pedagógico y lo artístico cuando ponemos en tensión los modos en los que el arte produce conocimientos?, ¿Qué función juega la Investigación Artística en la visualización de estos conocimientos?, ¿Cómo dar cuenta de un proceso artístico para que los conocimientos producidos puedan ser valorados y utilizados en la academia?

Durante mi investigación doctoral y, específicamente, a partir de la edición de los vídeos comprendidos en ésta, pude visibilizar nueve modos de conocer que los participantes de esta investigación y yo compartimos. Me di a la tarea de relacionar los conceptos de dichos modos para encontrar qué espacio social de producción de conocimiento se dibujó entre su proyecto artístico y mi investigación.

El espacio social de producción de conocimiento lo concibo como la materialización de las relaciones pedagógicas entre los sujetos implicados. Utilicé el concepto de espacio social ${ }^{5}$ de Henri Lefevbre para pesar tanto la producción artística como la producción de

\footnotetext{
${ }^{1}$ Atkinson 2014

2 Gergen y Gergen 2011

${ }^{3}$ Bryant y Charmaz 2013; Kelle 2013; Noerager Stern 2013

${ }^{4}$ Stronach et al. 2007; Macbeth 2001

${ }^{5}$ Lefebvre 2013
} 
conocimiento como generadoras de relaciones más que como fabricantes de objetos. El espacio social es la materialización de relaciones sociales (artísticas y pedagógicas) con el cual puede visibilizar y reconocer la forma del conocimiento del proyecto artístico. Así, el espacio social de producción de conocimiento al que apelé se constituyó mediante un acontecimiento pedagógico que generó un lugar (made place), una posibilidad que no había sido vislumbrada, para escenarios reflexivos. Ellsworth lo llamaría lugares anómalos de aprendizaje -anoumalous places for learning ${ }^{6}$ :

"Pedagogy is seldom engaged as an event in which the materiality of a time and place of learning impinges on the materiality of the learning self understood as a 'processual engagement of duration and movement, articulated through webs of sensations across landscapes and panoramas of spaces, bodies and time' (Kennedy 2003, p.4) and educational materials" ${ }^{7}$.

De esta manera, el espacio social resultado tanto de los vídeos como de mi tesis, entendidos como significaciones audiovisuales o textuales, no sólo produjo representaciones sino construyó nuevas subjetividades, nuevas formas de ser en relación ${ }^{8}$. Esto es, unas relaciones pedagógicas otras.

A través de las experiencias de mi investigación y a partir de las miradas de los otros (self-other), construí la posibilidad de desconocer mis modos de comprensión sobre cómo percibía el vínculo entre lo artístico, lo pedagógico y la producción de conocimiento. Concebía la producción de conocimiento como un proceso que tenía lugar en una persona, que, en cierto sentido, fabricaba un producto: obtenía sensaciones e información del mundo exterior, las procesaba individualmente según su contexto y experiencias anteriores, para después externalizarlo en forma de idea (como artefacto). Lo pedagógico lo entendía atado a lo educativo, por lo que aprendizaje y conocimiento me parecían sinónimos. Lo artístico lo sabía productor de conocimientos, pero no sabía cómo lo hacía. Extrañarme dentro de mis propios marcos de comprensión, implicó el tránsito que asumí al poner en marcha mi investigación. Y, el desplazamiento de unos modos de comprensión a otros, ha sido la huella que muestra la materialización de las relaciones pedagógicas que establecimos. Esto es, el espacio social de producción de conocimiento resultante de esta investigación, es decir, la investigación misma.

“Transitional space... exists always and everywhere as potential” "....assemblages that incorporate pedagogical pivot points create a fluid, moving pivot place that puts inside and outside, self and other, personal and social into relation" ${ }^{10}$. Basándome en las ideas de Ellsworth, el espacio social de producción de conocimiento podría ser este lugar pivote en movimiento (moving pivot place), que ensambla los modos de conocer que antes desarrollé. Un espacio transicional que activa nuevas posibilidades de conocer y de ser en relación.

Considero que la producción de estos conocimientos sirve de motor para seguir planteando otras preguntas desde una postura más comprometida y más congruente, pues como dice Benjamin "La mejor tendencia es falsa si no incluye el ejemplo de la actitud con la cual es posible seguirla. Y el escritor sólo puede ejemplificar esta actitud allí donde hace algo realmente: en su acción de escribir"11. Entonces, ¿cómo invito, desde mi proceso de investigación, desde mi producción visual y escrita, a posicionar a lo pares como colegas y colaboradores?

En el proceso de esta investigación he aprendido sobre algunos posibles modos de producción de conocimiento en un proyecto artístico. Ahora me pregunto: ¿cómo comparto este proceso de manera que se reactive en el espectador o en el lector, conseguir que se disemine? 0 , en términos de Deleuze y Guattari, para que genere hierba y no raíz ${ }^{12}$. Desde esa misma óptica que cuestiona la pasividad de diseminación de los conocimientos producidos, Barthes describe el placer que encuentra en la suspensión, momentánea, de la maquinaria de producción de conocimiento:

"as an intellectual pleasure that can be found in a temporary suspension of the repetitive machines of knowledge production, a pleasure resulting from hovering in the in-between, a brief balancing in the chasm or the fault of the feno- and the geno-text, producing as Barthes states, a 'staging of an appearance-as-disappearance ${ }^{\prime \prime 13}$

La Investigación Artística que realicé y de la que aquí he dado algunas pinceladas, se resiste a imponer una verdad sobre la manera de producir conocimientos en la investigación. Por eso, mi objetivo ha sido construir un aparato, una caja de herramientas, como diría Marrero ${ }^{14}$, que se pliegue y despliegue para desplazar estos relatos hacia otros campos de saber; un conjunto de posibles escenarios

\footnotetext{
${ }_{7}^{6}$ Ellsworth 2005, p.5

7 ibid., p. 24

8 Hernández y Padilla Petry 2011

${ }^{9}$ Ellsworth 2005, p. 32

10 ibid., p. 38

${ }^{11}$ Benjamin 2004, p. 49

${ }^{12}$ Deleuze y Guattari 2003

${ }_{13}$ Slager 2012, p. 77-78

${ }^{14}$ Marrero 2008
} 
que suspendan y rearticulen modos de conocer, y que, a su vez, desplieguen nuevas maneras de relacionarnos, de conocernos y de ser en conjunto.

\section{El espacio social de producción de conocimiento que se conforma al perturbar lo artístico y lo pedagógico.}

Al visibilizar y designar los nueve modos de conocer del proyecto La Síndrome de Sherwood delimité el espacio social de producción de conocimiento que conformamos entre el proyecto artístico y mi investigación. Delinear la constelación social-espacial-político que los nueve modos dibujaron cuestionó y reconfiguró otros posibles vínculos entre lo artístico y lo pedagógico en cuanto a la producción de conocimiento, la producción artística, la investigación y la conformación sujetos pedagógicos. Así, a partir de haber puesto en cuestión la Investigación Artística y la producción de conocimiento, esta investigación propone dar otro sentido al conocer a partir de la constelación social-político-espacial.

\section{Lo social}

Desde el posicionamiento que fue construyendo mi investigación resulta impensable la práctica artística y la producción de conocimiento como procesos individuales. $Y$ aún cuando esto no es nuevo -ejemplo de ello menciono al arte contemporáneo o posmoderno y teorías como la estética relacional ${ }^{15}$ - defiendo la práctica artística como una intervención en la red de significaciones ${ }^{16}$ y por tanto una relación social y epistemológica. Trabajar desde la dimensión social y epistemológica de un proyecto artístico me compromete a defender el proceso transitivo y ontológico, y rechazar la noción de obra de arte como resultado final.

Lo mismo pasa con la producción de conocimiento. Al valorar sus cualidades relacionales, transitivas y, sobretodo ontológicas, más que entenderla como fabricante de conocimientos objetivos y fijos, el vínculo entre sujetos, objetos y espacios se ilumina y, la relación pedagógica se asume como responsable de conocimientos relacionales, transitivos y ontológicos, que construyen redes de sujetos (pedagógicos) en construcción.

La colaboración que Núria Güell rescata como uno de sus modos de conocer, resonó en mi investigación hasta reconfigurar las relaciones que como investigadora establecí con los otros, que no los concebía más como sujetos de investigación ni como informantes ${ }^{17}$, sino como colaboradores. Pensar a los otros como colaboradores en la investigación requiere valorar los saberes de los otros y reconocerlos como productores de conocimientos. Así, si la investigación es capaz de mirar desde una perspectiva múltiple, podrá reconocer sus conocimientos relacionales, transitivos y ontológicos e integrarlos en la red de significaciones que teje una investigación, que entonces será social no por investigar a los sujetos desde un enfoque sociológico, sino por defender la investigación como un proceso colectivo y social.

Ahora, asumir esta postura social respecto a lo artístico, lo pedagógico y respecto a la investigación me compromete como investigadora a ser perceptiva papel activo de los pares, como resonancias de mi investigación; conocer a partir del feedback, diría Núria. Me parece fundamental el papel que los pares pueden desempeñar en una Investigación Artística donde los límites y los estatutos de rigor son tan variables. Los pares, entonces, serán quienes legitimen la validez de otras formas de investigar partir de la transparencia en la toma de decisiones (metodología). Reconocer el feedback de los pares como la legitimación de otras formas de conocer será establecer una co-relación de evaluación y validación entre compañeros.

\section{Lo político}

A medida que se fue desarrollando mi investigación, y conforme me acerqué a teorías pedagógicas como las de Atkinson y Ellsworth y construccionistas $^{18}$, comprendí que además de preguntarme qué conocimientos producimos, era mi responsabilidad como investigadora también preguntarme ¿a qué responden dichas significaciones?, ¿a quién benefician estos entendimientos?

Así que además de producir conocimientos y visibilizarlos, mi investigación habría de problematizarlos. Lo que Oriol argumentó con la idea de trabajar desde el conflicto, y Atkinson ha explicado como perturbar los marcos de entendimiento ${ }^{19}$, yo habría que hacerlo con las mismas representaciones y narrativas que estaba construyendo tanto en los vídeos como en el texto.

Consecuencia de ello fue el desplazamiento que sufrió mi pregunta de investigación de ¿cómo produce conocimientos un proyecto artístico?, a ¿cómo se modifican lo artístico y lo pedagógico a partir de una investigación que visibiliza y cuestiona los modos de conocer de un proyecto artístico?

\footnotetext{
${ }^{15}$ Bourriaud 2006

${ }^{16}$ Bourriaud 2005

17 Pink 2001

18 Gergen y Gergen 2011; Ibáñez Gracia 2001; İ̃niguez 2003

${ }^{19}$ Atkinson 2011
} 
Este desplazamiento marca un posicionamiento que, al entender lo epistemológico como político, supone irrumpir en el tipo de conocimientos que busca y promueve. $Y$ es a partir de generar conocimientos disruptivos que la práctica artística al igual que la investigación, puede ser política, no por el tema que trate sino por la manera de problematizar la situación y de cuestionar 'lo dado'. Así, pienso que el proyecto de Núria no es político por lidiar con tema de la policía sino por la postura con la que se acerca a esta situación, la desconoce y la cuestiona; para usar sus mismas palabras, en cómo la interpela.

Pero, es necesario dejar en claro que no es mi intención establecer dualidades entre lo normativo y lo alternativo. Y para salir de esta trampa dualista recurro al concepto de self-other ${ }^{20}$ que mira nuestros propios marcos de entendimiento con extrañeza para encontrar en nuestra propia perspectiva o posicionamiento estas normativas que des-conocer. La reflexividad me permitió cuestionar mis normativas en las representaciones que fui construyendo y des-conocer mis posicionamientos sobre lo artístico, lo pedagógico, a través de lo político.

\section{Lo espacial}

Lo espacial es una inquietud en mi hacer que ya se manifestaba en mis producciones artísticas ${ }^{21}$. Primero el espacio urbano, y después eso conformó un interés más sustancioso a partir del concepto de espacio social. Así mismo, mis visiones de del espacio también se modificaron según el desarrollo de esta investigación. Primero que nada comprender la posibilidad de disociar lo educativo de lo pedagógico ${ }^{22}$. Para después, ubicar las relaciones pedagógicas fuera de los límites escolares que limitan al sujeto a dos únicas posturas, y comprender cualquier sitio como una posibilidad espacial para construir conocimientos y una posibilidad epistemológica para construir espacios pedagógicos.

Lo artístico y lo pedagógico fungen como productores de conocimientos espaciales: corporeizados, situados y contextualizados, es decir que se significan en relación con los espacios-cuerpos. Éstas no son prácticas aisladas ni fijas. Están en constante transformación epistemológica tanto espacios como sujetos; son conocimientos transitivos y ontológicos, pero situados, contextualizados y corporeizados.

A partir de desvelar los nueve modos de conocer de Núria y Oriol pude visibilizar cómo la producción de conocimiento construye espacios: el proyecto artístico se volvió un espacio de reflexión y aprendizajes y construyó sujetos pedagógicos: la artista, el comisario y la investigadora, todos estábamos explorando y aprendiendo de nuestras formas de conocer a partir del arte y la investigación.

En el vídeo, Oriol Fontdevila explica cómo en su práctica comisarial busca no obviar los límites con los que trabaja sino activarlos a partir de convertirlos en una bisagra. Yo entiendo esta bisagra como una herramienta pedagógica que, en términos de Deleuze, puede convertir una línea, en un pliegue; la unidad o lo dual en múltiple ${ }^{23}$, que puede proyectar una situación hacia otras dimensiones, hacia otro terrenos con distintos contextos, otros cuerpos y por tanto otros marcos de entendimiento. Es decir que la bisagra puede generar, tanto en lo artístico como en lo educativo, reflexividad. La reflexividad moverá a los conocimientos no de lugares físicos sino los marcos de interpretación para desconocer lo ya sabido y volver a explorar desde la mirada de otro sujeto en nuestro mismo cuerpo, un sujeto pedagógico.

\section{Conclusiones}

Irrumpir las nociones de investigación, de lo artístico y lo pedagógico, ha sido el detonador para engarzar otros escenarios de significación y generar nuevas posibilidades de conocer, de nombrar y de ser. Al tiempo que en mi investigación doctoral se construyó un espacio social de producción de conocimiento, también nos fuimos construyendo como una red de sujetos pedagógicos en transición.

Des-conocer los mismos marcos de comprensión que fui generando a lo largo de mi investigación, abrió espacios disruptivos que desestabilizaron lo que en el proceso se había vuelto establecido, y, ahí fue que se posibilitó la emergencia de nuevos espacios de conocimiento. En este caso a través de la Investigación Artística rompí entre el arte e investigación (universidad) lo estipulado como proceso de investigación (rigor, transparencia, validación de datos, metodología) para posibilitar nuevas relaciones y nuevos escenarios entre lo artístico y lo pedagógico.

Los nueve modos de conocer que el vídeo muestra sobre el proyecto de Núria y Oriol ejemplifican otras formas por medio de las cuales el saber puede ser forjado y cuestionado a través del arte. Y a través de éste conformar nuevos sujetos pedagógicos engarzados en una red epistemológica consciente y comprometida.

\footnotetext{
${ }^{20}$ Atkinson 2014

${ }^{21}$ Ver htttp://www.natalia-calderon.com

${ }^{22}$ Hernández 2006

${ }^{23}$ Deleuze 1989
} 


\section{FUENTES REFERENCIALES}

ATKINSON, D. 2011. Art, equality and learning: pedagogies against the state. Rotterdam: Sense.

ATKINSON, D., 2014. The Adventure of Pedagogy, Learning and the Not-Known. Seminario en el programa de postgrado Artes y Educación. Sección de Pedagogías culturales, Facultad de Bellas Artes. Universidad de Barcelona.

BENJAMIN, W. 2004. El autor como productor. México, D.F: Itaca.

BOURRIAUD, N. 2005. Postproducción. Buenos Aires: Adriana Hidalgo Editora.

BOURRIAUD, N. 2006. Estética relacional. Buenos Aires: Adriana Hidalgo.

BRYANT, A. y CHARMAZ, K. 2013. Introduction Grounded Theory Research: Methods and Practices. En: A. BRYANT y K. CHARMAZ (eds.), The SAGE Handbook of Grounded Theory. London; Thousand Oaks, Calif: SAGE, pp. 1-28.

DELEUZE, G. 1989. El Pliegue: [Leibniz y el barroco]. Barcelona [etc.]: Paidós.

ELLSWORTH, E. 2005. Places of Learning: media, architecture, pedagogy. 1. New York and London: Routledge Falmer.

GERGEN, J.K. y GERGEN, M. 2011. Reflexiones sobre la construcción social. $1^{\circ}$. Madrid: Paidós.

HERNÁNDEZ, F. 2006. Campos, temas y metodologías para la investigación relacionada con las artes. En: INSTITUTO SUPERIOR DE FORMACIÓN DEL PROFESORADO, Bases para un debate sobre investigación artística. Madrid: Secretaría General Técnica. Subdirección General de Información y Publicaciones.

HERNÁNDEZ, F. y PADILLA PETRY, P. 2011. De investigador sobre jóvenes a investigar con jóvenes: relato de un proceso. I Jornadas "Investigar con los jóvenes: cuestiones temáticas, metodológicas, éticas y educativas. Barcelona: s.n.

IBÁÑEZ GRACIA, T. 2001. Municiones para disidentes: realidad-verdad-política. Barcelona: Gedisa. Cla-de-ma.

ÍÑIGUEZ, L. 2003. La psicología social en la encrucijada postconstruccionista. Historicidad, subjetividad, performatividad, acción. XII Encontro Nacional da ABRAPSO. Estratégias de invenção - a Psicologia Social no contemporâneo. Porto Alegre: Pontifícia Universida de Católica do Rio Grande do Sul.

KELLE, U. 2013. The Development of Categories: Different Approaches in Grounded Theory. En: A. BRYANT y K. CHARMAZ (eds.), The SAGE Handbook of Grounded Theory. London ; Thousand Oaks, Calif: SAGE, pp. 191-213.

LEFEBVRE, H. 2013. La producción del espacio. Madrid: Capitán Swing.

MACBETH, D. 2001. On «Reflexivity in Qualitative Research: Two Readings, and a Third ; 7; 35. Qualitative Inquiry. S.I.: Sage, pp. 3568.

MARRERO GUILLAMÓN, I. 2008. La fábrica del conflicto. Terciarización, lucha social y patrimonio en Can Ricart, Barcelona. [Consulta: 15 septiembre 2014]. Disponible en: http://www.tdx.cat/handle/10803/722.

NOERAGER STERN, P. 2013. On Solid Ground: Essential Properties for Growing Grounded Theory. En: A. BRYANT y K. CHARMAZ (eds.), The SAGE Handbook of Grounded Theory. London ; Thousand Oaks, Calif: SAGE Publications, pp. 114-126.

PINK, S. 2001. Doing Visual Ethnography. London: Sage.

SLAGER, H. 2012. The Pleasure of Research. 1. Helsinki: Finnish Academy of Fine Arts.

STRONACH, I., GARRATT, D., PEARCE, C. y PIPER, H. 2007. Reflexivity, the Picturing of Selves, the Forging of Method. Sage Publications, vol. 13 , no. 2 , pp. 179-203. 